
alcohol en adolescentes y adultos jóvenes. Cuadernos de Psicología del Deporte, 19(3), 24-36

\title{
Compromiso con el deporte y su relación con el consumo de alcohol en adolescentes y adultos jóvenes
}

\section{Engagement to sports and its relationship with alcohol consumption in adolescents and young adults}

\section{Compromisso com o esporte e sua relação com o consumo de álcool em adolescentes e adultos jovens}

\author{
Vanina Schmidt ${ }^{123}$; Ignacio Celsi ${ }^{2}$; María Fernanda Molina ${ }^{24}$; María Julia Raimundi ${ }^{123}$; María García- \\ Arabehety $^{2}$; Mauro Pérez-Gaido ${ }^{2}$; Darío Iglesias ${ }^{2}$; María Alejandra Gonzalez ${ }^{2}$ \\ ${ }^{1}$ Consejo Nacional de Investigaciones Cientificas y Técnicas (CONICET), Argentina; ${ }^{2}$ Instituto de \\ Investigaciones, Facultad de Psicología, Universidad de Buenos Aires, Argentina; ${ }^{3}$ Facultad de \\ Psicología, Universidad Abierta Interamericana, Argentina; ${ }^{4}$ Universidad Nacional Tres de Febrero, \\ Argentina
}

\begin{abstract}
RESUMEN
El consumo de alcohol representa uno de los problemas más graves de salud pública a nivel global. Si bien se señala al deporte como estrategia mundial efectiva para amortiguar el impacto de esta problemática, los estudios han concluido que no es el deporte per se sino aspectos motivacionales asociados al mismo los que podrían tener efectos protectores. El compromiso con el deporte no ha sido investigado como posible factor protector del consumo de alcohol. Objetivo: Indagar la relación entre el compromiso con el deporte y el consumo de alcohol. Método. Participantes: 362 jóvenes (15 a 29 años) deportistas de Argentina. Instrumentos: Cuestionario sociodemográfico, Cuestionario de Compromiso con el Deporte (AEQ), Cuestionario de Identificación de los Trastornos Debidos al Consumo de Alcohol (AUDIT-C). Procesamiento: Se realizó análisis de varianza y regresión logística con el paquete SPSS v.21. Resultados: los deportistas consumen tanto como los no deportistas, pero quienes presentan mayor energía invertida en su deporte, presentan una menor frecuencia de consumo actual y de consumo excesivo. Se discuten las implicancias prácticas de estos resultados.
\end{abstract}

Palabras clave: Compromiso - Deporte - Alcohol - Factores protectores.

\section{ABSTRACT}

Alcohol consumption represents one of the most serious public health problems worldwide. Although sport is indicated as an effective global strategy to cushion the impact of this problem, studies have concluded that it is not sport per se, but motivational aspects associated with it, that could have protective effects. The engagement with sport has not been investigated as a possible protective factor for alcohol consumption. Aim: To investigate the relationship between engagement with sports and alcohol consumption. Method. Participants: 362 young people (15 to 29 years old) athletes from Argentina. Instruments: Socio-demographic questionnaire, Sports Engagement Questionnaire (AEQ), Alcohol Use Disorders Identification Test (AUDIT-C). Processing: Analysis of variance and 


\section{Compromiso con el deporte y su relación con el consumo de alcohol}

logistic regression were performed with the SPSS package v.21. Results: athletes consume as much as non-athletes but those who have more energy invested in their sport, have current and excessive consumption less frequently. The practical implications of these results are discussed.

Keywords: Engagement - Sport - Alcohol - Protective factors.

\section{RESUMO}

O consumo de álcool representa um dos mais sérios problemas de saúde pública no mundo. Embora o esporte seja apontado como uma estratégia global eficaz para amortecer o impacto desse problema, estudos concluíram que não é o esporte per se, mas os aspectos motivacionais associados a ele, que poderiam ter efeitos protetores. O compromisso com o esporte não tem sido investigado como um possível fator de proteção contra o consumo de álcool. Objetivo: Investigar a relação entre compromisso com esporte e consumo de álcool. Método Participantes: 362 jovens (15 a 29 anos) atletas da Argentina. Instrumentos: Questionário sociodemográfico, Questionário de Compromisso com o Esporte (AEQ), Questionário para Identificação de Distúrbios Devido ao Consumo de Álcool (AUDIT-C). Processamento: A análise de variância e a regressão logística foram realizadas com o pacote SPSS v.21. Resultados: os atletas consomem tanto quanto os não atletas, mas aqueles que têm mais energia investida em seu esporte, apresentam menor frequência de consumo atual e consumo excessivo. As implicações práticas desses resultados são discutidas.

Palavras chave: Compromisso - Esporte - Álcool - Fatores de proteção.

\section{INTRODUCCIÓN}

La investigación en consumo de alcohol es hoy una tarea básica necesaria para el diseño de Políticas Públicas. Para la Organización Mundial de la Salud (OMS, 2014), el uso nocivo de esta sustancia es responsable a nivel mundial del $5,9 \%$ de todas las muertes y representa el 5,1\% de la carga mundial de morbilidad. Es también señalada como la principal causa de traumatismos involuntarios por lesiones, trastornos neuropsiquiátricos, depresión, malnutrición e importante factor de riesgo de enfermedades hepáticas, cardiovasculares, diversos tipos de cáncer, úlceras, anemia, coagulación defectuosa y de enfermedades de transmisión y funcionamiento sexual (OMS, 2014).

En Iberoamérica, el patrón más preocupante es el consumo episódico excesivo de alcohol (CEEA) definido como la ingesta de gran cantidad de alcohol (aproximadamente más de 5 Unidades de Bebidas [UBs] $=60 \mathrm{gr} / \mathrm{cc}$ alcohol puro) en una sola ocasión o lapso breve ( 3 a $4 \mathrm{hs}$ ). Este es el patrón de mayor impacto en el sistema sociosanitario y se afirma que una de cada cinco muertes de jóvenes está relacionada con el mismo (OMS, 2010). Esta modalidad de ingesta ha aumentado en pocos años en un $300 \%$ en mujeres y $50 \%$ en varones de las
Américas (Organización Panamericana de la Salud [OPS], 2015).

En Argentina, la ingesta de alcohol se ubica tras el tabaco, como la segunda causa de muerte asociada al consumo de sustancias psicoactivas (OAD, 2017a). Al mismo tiempo, los estudios realizados en territorio nacional muestran una situación alarmante: el consumo de alcohol aumentó en los últimos años, cada vez más mujeres consumen y cada vez se consume a edades más tempranas (OAD, 2014; OPS, 2015; Secretaría de Políticas Integrales sobre Drogas de la Nación Argentina [SEDRONAR] 2017b). Los niveles más altos de consumo se observan en jóvenes de 18 a 24 años (SEDRONAR, 2017a). Y si bien los jóvenes que abarcan esta franja etaria son señalados como los que mayor riesgo presentan a la hora de consumir alcohol, preocupa especialmente la etapa adolescente (12-18 años).

Los últimos resultados obtenidos a nivel poblacional (12 a 65 años) registran para la población adolescente el mayor aumento sobre los niveles de consumo de alcohol durante el periodo 2010-2017 (más de 13 puntos porcentuales) (SEDRONAR, 2017b). Asimismo, los resultados de este estudio indican que resulta más frecuente hallar un consumo problemático y excesivo dentro de la población joven (12 a 24 años) (SEDRONAR, 2017a). Por otra parte, 


\section{Schmidt, V., Celsi, I., Molina, M.F., Raimundi, M.J., García-Arabehety,M., Pérez-Gaido, M., Iglesias, D., \& González, M.A.}

se afirma que uno de cada dos niños y adolescentes que consumieron alcohol en el último mes lo hizo de forma abusiva (SEDRONAR, 2017c).

Este escenario obliga a persistir en los esfuerzos por identificar aspectos en la vida de los jóvenes que contribuyen a un menor consumo de alcohol y a un desarrollo positivo.

Desde el contexto investigativo, se encuentra claramente establecida la contribución del ejercicio físico y la práctica deportiva a un estilo de vida saludable (Balaguer, 2002; Castillo, Balaguer y García-Merita, 2007), así como sus efectos favorables para un autoconcepto positivo y el desarrollo de la autoestima en población joven (Balaguer, Atienza y Duda, 2012). A su vez, desde los programas de promoción de la salud y de prevención, se intenta favorecer el desarrollo deportivo de la población joven como parte de las estrategias para paliar el uso nocivo de alcohol (OMS, 2004; OPS, 2010; UNODC, 2014). Sin embargo, los estudios que intentan mostrar que el deporte per se tiene efectos positivos sobre el consumo excesivo de alcohol han arrojado resultados inconsistentes.

Ceschini et al. (2015) encontraron en jóvenes brasileños que la prevalencia de actividad física se asocia a un menor consumo de bebidas alcohólicas. En consonancia con este resultado, otros autores trabajando con muestras españolas han encontrado asociaciones negativas entre la práctica de actividad física-deportiva y el consumo de alcohol en población adolescente (Castillo, Balaguer y GarcíaMerita, 2007; Latorre-Román, Cámara-Pérez y García-Pinillos, 2014; Pastor, Balaguer, y GarcíaMerita, 2006; Ruiz-Juan y Ruiz-Risueño, 2011), es decir, observan que en dicha población a mayor actividad física hay menor consumo de alcohol. Sin embargo, algunos estudios no han encontrado una asociación entre la práctica físico-deportiva y el consumo de alcohol (Castillo, Balaguer y GarcíaMerita, 2007; Ferriz, 2014; Jiménez et al., 2008; Moore, 2005; Vaquero-Cristóbal, Isorna y Ruiz, 2012) e, incluso, otros estudios permiten concluir que tal práctica se asocia positivamente a una mayor prevalencia de consumo de alcohol, mayor frecuencia de consumo actual y mayor frecuencia de CEEA (Bedendo y Noto, 2015; Lorente et al., 2004).

Varios son los autores que sostienen que la participación en el deporte no es en sí misma positiva o negativa. Son más bien las circunstancias que la rodean y la forma en la que la definen los otros significativos, lo que afecta la percepción que tienen las personas sobre la práctica físico-deportiva $\mathrm{y}$, en consecuencia, lo que resulta en una experiencia de bienestar o malestar (Balaguer,Castillo y Duda 2008; Bedendo y Noto, 2015; Hedstrom y Gould, 2004; Jones y Paulhus, 2011). Diversos estudios han mostrado la importancia que adquieren los procesos motivacionales en la práctica físico-deportiva (e.g.; Ceschini et al., 2015; Coterón, Sampedro, Franco y Pérez-Tejero, 2013; Duda, 2013; Ferriz, 2014).

Uno de los principales marcos teóricos desde los que se ha abordado el estudio de dichos procesos es la Teoría de la Autodeterminación (SDT; Deci y Ryan, 1985). La conceptualización original de Deci (1975) plantea que las personas pueden presentar dos tipos de motivación para realizar cualquier actividad: la motivación intrínseca, mediante la cual la conducta se lleva a cabo por la propia satisfacción en su realización y la motivación extrínseca, en la que el motivo de realización está promovido por una recompensa o beneficio por dicho comportamiento.

Asimismo, desde esta teoría se postula que las personas cuando interaccionan con su ambiente necesitan sentirse competentes, autónomas y relacionadas con los demás, y que la satisfacción de estas necesidades es esencial para el desarrollo tanto de la motivación intrínseca o autodeterminada, como del bienestar psicológico (Ryan y Deci, 2000). En la satisfacción o frustración de estas necesidades el ambiente social juega un importante papel y más concretamente los climas motivacionales creados por las figuras de autoridad (entrenadores, padres, pares) (Balaguer et al. 2008).

La motivación intrínsecamente determinada constituye la regulación autónoma de la motivación que lleva al compromiso con una actividad. Es decir, los deportistas que están intrínsecamente motivados participan en su deporte debido a los sentimientos de diversión, interés y satisfacción que son inherentes a esta actividad (Deci y Ryan, 1985). En este sentido, se plantea que la satisfacción de las necesidades básicas constituye un antecedente para el desarrollo del compromiso con el deporte (Hodge, Lonsdale y Jackson, 2009).

El compromiso (engagement) es un proceso cognitivo-afectivo persistente y positivo caracterizado por la creencia en la propia capacidad para lograr un nivel de rendimiento y conseguir metas (confianza), el deseo de invertir esfuerzo y 


\section{Compromiso con el deporte y su relación con el consumo de alcohol}

tiempo en el logro de objetivos importantes para uno (dedicación), el vigor o vivacidad física, mental y emocional (energía) y la sensación de disfrute (entusiasmo) (Lonsdale, Hodge y Raedeke, 2007). El compromiso emerge en la literatura como un constructo de orden superior, que engloba diferentes conceptos (e.g. disfrute, vitalidad, autoconfianza, pasión, etc.), permitiendo así comprender la complejidad de las experiencias positivas en el deporte (Lonsdale et al., 2007).

El presente estudio intenta responder cómo se relaciona el compromiso con el deporte y el consumo de alcohol. Específicamente, busca identificar los aspectos del compromiso con el deporte (confianza, dedicación, energía y entusiasmo) que podrían predecir menor frecuencia de consumo actual de alcohol, y menor prevalencia y frecuencia de consumo episódico excesivo de alcohol.

En términos de la SDT, un clima motivacional positivo se asocia a mayor compromiso con el deporte (Coterón et al., 2013). A su vez, aquellos jóvenes motivados intrínsecamente consumen alcohol con menos frecuencia, menos cantidad $y$ experimentan atracones con menor frecuencia (Rockafellow y Saules, 2006). Un estudio realizado con 513 deportistas españoles (16 a 58 años) reveló que la orientación al ego (el éxito percibido cuando se supera a los demás, en contraposición a la orientación a la tarea cuya meta es la mejora personal) tiene claros efectos sobre un mayor consumo de alcohol y que la motivación autodeterminada se asocia de manera moderada a menor consumo de dicha sustancia (Moreno-Murcia, González-Cutre Coll y Cervello-Gimeno, 2008). Otro estudio con 626 deportistas deportistas españoles (14 a 19 años) concluyó que las relaciones entre motivación intrínseca hacia la práctica, aprendizaje y perfeccionamiento se asocia con menor prevalencia de consumo de alcohol, pero estas asociaciones fueron débiles aun cuando se confirman a través de un modelo de ecuaciones estructurales (Usán y Salavera, 2017).

Dado estos antecedentes, se hipotetiza que los jóvenes con niveles de compromiso más elevados presentan menor probabilidad de una alta frecuencia de consumo actual (hipótesis 1), menor probabilidad de un patrón de tipo CEEA (hipótesis 2) y menor probabilidad de una alta frecuencia de CEEA (hipótesis 3).

\section{MATERIAL Y MÉTODOS}

\author{
Alcance del estudio y diseño
}

Estudio descriptivo-correlacional de diseño no experimental transversal (Hernández Sampieri, Fernández-Collado y Baptista Lucio, 2014).

\section{Participantes}

Colaboraron 362 jóvenes (57.2\% género masculino, $42.8 \%$ género femenino) de entre 15 y 29 años $(M=$ 18.2; $D T=3$ ) que formaban parte de distintos equipos pertenecientes a clubes deportivos del Área Metropolitana de Buenos Aires (AMBA). Los deportes que practicaban eran: fútbol 11 , fútbol sala, básquetbol, voleibol, balonmano, rugby y hockey sobre césped. La mayoría (72\%) tenía una alta frecuencia de entrenamiento (tres veces por semana o más) y competía semanalmente (93\%).

\section{Instrumentos}

- Cuestionario sociodemográfico. Diseñado ad-hoc para caracterizar a los participantes en función de variables sociodemográficas consideradas relevantes: género, edad, nivel educativo y composición familiar).

- Cuestionario de situación con la actividad físicodeportiva. Diseñado ad-hoc para evaluar tipo de actividad, antigüedad en el deporte, frecuencia semanal de entrenamiento y de competencia, horas de práctica, nivel de competición o torneo $\mathrm{y}$ participación en Selecciones Nacionales.

- Cuestionario de compromiso en deportistas (Atlhete Engagement Questionnaire [AEQ] de Lonsdale, Hodge y Jackson, 2007; adaptación: Raimundi, Molina, Schmidt y Lonsdale, en preparación). Evalúa cuatro dimensiones del compromiso (confianza, dedicación, energía y entusiasmo) mediante 16 ítems con cinco opciones de respuesta que estiman la frecuencia con la que el deportista percibe esos componentes del compromiso. Posee adecuados indicadores de validez y confiabilidad en su versión local. Para la presente muestra, la consistencia interna para Confianza fue de .86, para Energía fue de .80, para Dedicación fue de $.81 \mathrm{y}$ para Entusiasmo fue de .85 . 


\section{Schmidt, V., Celsi, I., Molina, M.F., Raimundi, M.J., García-Arabehety,M., Pérez-Gaido, M., Iglesias, D., \& González, M.A.}

- Cuestionario de Identificación de los Trastornos Debidos al Consumo de Alcohol versión abreviada (AUDIT-C de Bush, Kivlahan, McDonell, Fihn y Bradley, 1998; basado en el AUDIT de Saunders, Aasland, Babor, de la Fuente y Grant, 1993; adaptación local: Cremonte, Ledesma, Cherpitel y Borges, 2010). El AUDIT es un test de tamizaje para la identificación de consumo moderado, de riesgo, perjudicial y posible dependencia. Contiene 10 preguntas con escala Likert de 5 puntos. Los tres primeros ítems forman parte del AUDIT-C y miden frecuencia de consumo actual, cantidad y frecuencia de consumo episódico excesivo. Para medir cantidad se le pide al sujeto que indique la cantidad de bebidas estándar consumidas en la misma ocasión. Cada bebida estándar (UB) equivale a $330 \mathrm{~cm} 3$ de cerveza, un vaso de vino o una medida de bebida blanca. Se calcula la UB teniendo en cuenta los gramos de alcohol puro ingeridos por un sujeto en la misma ocasión. A partir del punto de corte, se considera que la persona realiza un consumo episódico excesivo de alcohol definido como la ingesta de gran cantidad de alcohol (más de 4/5 UBs $=60 \mathrm{Gr} / \mathrm{cc}$ alcohol puro aproximadamente) en una sola ocasión y en un lapso corto (3 o 4 horas). Se ajustó el punto de corte en función del género (mujeres: 4 UB y varones: $5 \mathrm{UB}$ ) de acuerdo a lo establecido por la literatura. El AUDIT-C mostró alta sensibilidad y baja especificidad, diferencias en el punto de corte óptimo en función del género, relativa consistencia interna y alta estabilidad test-retest en población argentina (Conde, Gimenez y Cremonte, 2018). Para la presente muestra, la consistencia interna por Alpha de Cronbach fue de .85 .

\section{Procedimiento}

Se contactó a entrenadores de distintos deportes colectivos, pertenecientes a cinco clubes del AMBA para poder aplicar el protocolo a jóvenes deportistas que realizan de forma amateur su actividad deportiva de equipo. Se solicitó un consentimiento informado por escrito a los deportistas o sus padres (en caso de ser menores de edad). El protocolo fue aplicado en el horario de entrenamiento, coordinando previamente los momentos disponibles con sus entrenadores. Los participantes se comprometieron a contestar de manera honesta y anónima. Luego del procesamiento inicial de los datos, los participantes y entrenadores recibieron un informe, a modo de devolución, con los principales resultados obtenidos para cada equipo. Se han respetado los aspectos éticos y deontológicos locales e internacionales. Se cuenta con el aval del comité de ética.

\section{Análisis estadístico}

Se realizó un análisis descriptivo de cada una de las variables que componen el estudio y un ANOVA (con un nivel de significación del 95\%) para conocer los efectos de los componentes del compromiso sobre las tres variables de consumo (frecuencia, cantidad y frecuencia de CEEA). Se utilizó el análisis de regresión logística (método: Introducir) para probar el poder predictivo del compromiso sobre las tres variables del AUDIT-C, Debido a que se ha documentado una relación entre el género y la edad con el consumo de alcohol, se estudió el poder predictivo de dichas variables sobre las variables dependientes. El género no resultó significativo para predecir frecuencia de consumo actual $\left(X_{2}[1,353]=\right.$ $.313 ; p=.57)$, cantidad de alcohol consumido $\left(X_{2}[1\right.$, $353]=.317 ; p=.07)$ y frecuencia de CEEA $\left(X_{2}[1\right.$, $254]=.758 ; p=.38)$ por lo que no se incluyó en los subsiguientes modelos. La edad solo fue significativa respecto de la frecuencia de consumo actual $\left(X_{2}[1\right.$, $353]=7.770 ; p=.011)$ y no fue significativa para cantidad de alcohol consumido $\left(X_{2}[1,353]=1.908\right.$; $p=.16)$ y frecuencia de CEEA $\left(X_{2}[1,254]=.758 ; p\right.$ $=.28$ ). Por este motivo, para el primer modelo se realizó un análisis estratificado en el que se incluyó la variable edad en el primer paso y la variable energía en el segundo paso (la única variable que resultó significativa en el ANOVA). Así, se testeó la capacidad de la variable energía para predecir frecuencia de consumo actual (baja: una vez al mes o menos vs. alta: dos veces por semana o más). En el segundo modelo, la capacidad de la variable energía para predecir cantidad de alcohol consumido en la misma ocasión (baja: menos de 4/5 UBs vs. alta: 4/5 UBs o más) y en el tercer modelo, la capacidad de esta variable para predecir frecuencia de CEEA (baja: menos de una vez al mes vs. alta: una vez al mes o más). Se eligió la regresión logística debido a que si bien el AUDIT-C posee una escala Likert de cinco puntos, el nivel de medición es ordinal y no presenta una distribución normal. A diferencia de la regresión lineal, en la regresión logística no es requisito que la variable dependiente tenga una distribución normal ni tampoco se realizan asunciones sobre las variables predictoras por lo que es un método más robusto que 


\section{Compromiso con el deporte y su relación con el consumo de alcohol}

la regresión lineal o el análisis discriminante (Bewick, Cheek, y Ball, 2005). Las variables dependientes del presente estudio (preguntas 1, 2 y 3 del AUDIT) fueron dicotomizadas en todos los casos porque lo que se busca a través de la regresión logística es pronosticar la probabilidad de que ocurra o no un determinado suceso (en este caso, la pertenencia a uno de los dos grupos de comparación) como función de ciertas variables que se presumen relevantes. La calidad de la predicción lograda por cada uno de los modelos se midió por medio del porcentaje de coincidencia entre el valor observado y el valor esperado por la predicción (es decir, la

\section{RESULTADOS}

Respecto del consumo de alcohol, el 84\% declaró consumir esta sustancia $(22 \%$ una vez al mes o menos, $44 \%$ de dos a cuatro veces por mes, $16 \%$ de dos a tres veces por semana y el $2 \%$ casi todos los días), el $34 \%$ de ellos presentó un CEEA y el 17\% presentó este patrón una vez por semana o más. Estos cantidad de aciertos). Se tomaron como medidas del tamaño del efecto los R2 de Cox \& Snell y Nagelkerke para conocer el porcentaje de varianza explicado por el modelo, más allá de la cantidad de aciertos en la predicción (Bewick et al., 2005). Sin embargo, sólo se toma para su interpretación el $R^{2}$ de Nagelkerke debido a que es una corrección del $R^{2}$ de Cox y Snell que adopta un valor máximo de 1, lo que facilita su interpretación. Se identificaron las variables con más relevancia a través del coeficiente $B$ y su significación. Se utilizó el paquete estadístico SPSS v. 21.

Tabla 1. Estadísticos descriptivos para los componentes de compromiso

\begin{tabular}{cccccc}
\hline $\begin{array}{c}\text { Muestra total } \\
(n=359)\end{array}$ & $M(D T)$ & Moda & Mediana & Asimetría & Curtosis \\
\hline Confianza & $14.99(3.15)$ & 16 & 15 & -.42 & -.25 \\
Energía & $16.43(2.43)$ & 16 & 16 & -.35 & -.11 \\
Dedicación & $16.78(2.62)$ & 20 & 17 & -.72 & .34 \\
Entusiasmo & $17.93(2.12)$ & 20 & 19 & -1.07 & 1.57 \\
\hline
\end{tabular}

Respecto del compromiso, se presentan los estadísticos descriptivos de la muestra total para los cuatro componentes de la variable evaluada (Tabla 1). resultados respecto del consumo de alcohol son similares a los reportados por estudios epidemiológicos nacionales con muestras de jóvenes población general (SEDRONAR, 2017a).

Tabla 2. Estadísticos descriptivos y ANOVA para compromiso, en función de la modalidad de consumo de alcohol

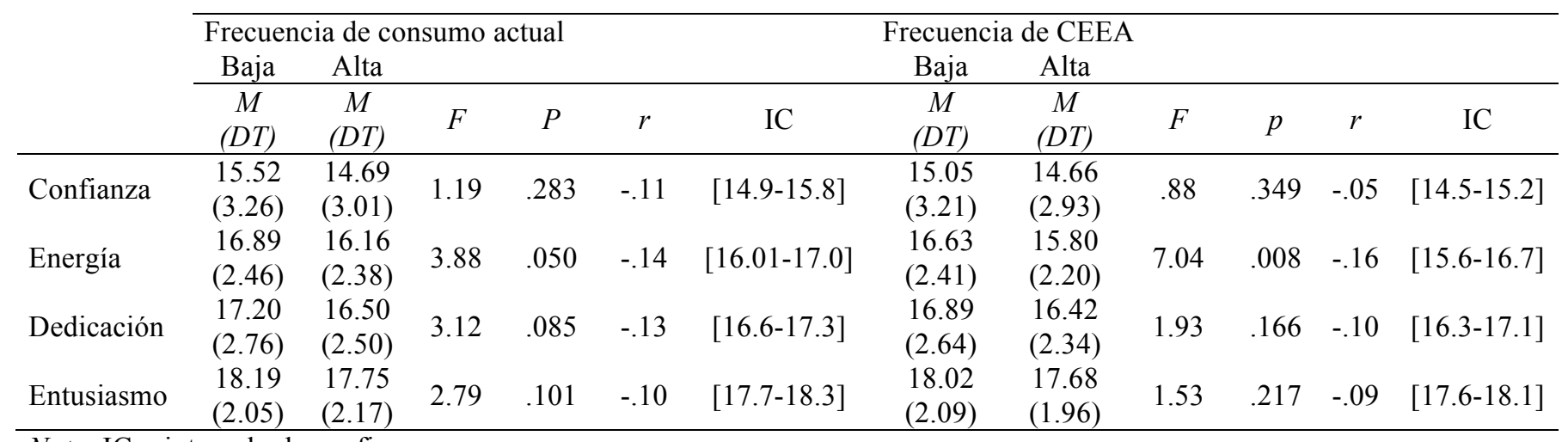

Nota: $\mathrm{IC}=$ intervalo de confianza

El análisis de la varianza (ANOVA) muestra que, por un lado, la energía es mayor entre quienes consumen de manera infrecuente comparado con quienes lo 


\section{Schmidt, V., Celsi, I., Molina, M.F., Raimundi, M.J., García-Arabehety,M., Pérez-Gaido, M., Iglesias, D., \& González, M.A.}

hacen frecuentemente $(p=.050) \mathrm{y}$, por otro lado, dicho componente es mayor entre quienes realizan con baja frecuencia CEEA comparado con los de alta frecuencia de CEEA $(p=.008)$. Por último, se muestra el tamaño del efecto, estudiado con el coeficiente $r$ de Pearson, para los componentes de compromiso en función de la modalidad de consumo de alcohol. Se observa que en todos los casos el tamaño del efecto es bajo (Tabla 2). Ningún componente del compromiso tuvo efectos en la cantidad de consumo de alcohol por ocasión.

Tabla 3. Capacidad predictiva de la energía respecto de la frecuencia de consumo actual de alcohol

$* p<.05$

Nota. $O R=$ odd ratios; $\mathrm{IC}=$ intervalo de confianza

\begin{tabular}{|c|c|c|c|c|c|c|c|}
\hline \multicolumn{8}{|c|}{ Modelo. VD: Frecuencia de consumo actual } \\
\hline \multicolumn{3}{|c|}{ Likelihood ratio test } & \multicolumn{3}{|c|}{$R^{2}$ Cox Snell } & \multicolumn{2}{|c|}{$R^{2}$ Nagerlkerke } \\
\hline$x^{2}$ & $G l$ & $p$ & \multirow{2}{*}{\multicolumn{3}{|c|}{.04}} & \multirow{2}{*}{\multicolumn{2}{|c|}{.03}} \\
\hline $7.48^{*}$ & 1 & .006 & & & & & \\
\hline Capacidad predictiva (\%) & & $\begin{array}{c}\text { Global } \\
64\end{array}$ & & \multicolumn{2}{|c|}{$\begin{array}{c}\text { Baja frecuencia } \\
17.3\end{array}$} & \multicolumn{2}{|c|}{$\begin{array}{l}\text { Alta frecuencia } \\
91\end{array}$} \\
\hline Variables en la ecuación & $B$ & $E T$ & Wald & $G l$ & $P$ & $O R$ & $95 \%$ IC OR \\
\hline Edad & $-0.99^{*}$ & 0.03 & 6.49 & 1 & .011 & 1.10 & [1.02-1.19] \\
\hline Energía & $-0.12^{*}$ & 0.05 & 6.12 & 1 & .013 & 0.89 & [0.81-0.97] \\
\hline \\
\hline \multicolumn{3}{|c|}{ Likelihood ratio test } & \multicolumn{3}{|c|}{$R^{2}$ Cox Snell } & \multicolumn{2}{|c|}{$R^{2}$ Nagerlkerke } \\
\hline$x^{2}$ & $G l$ & $P$ & & & & & \\
\hline $4.41 *$ & 1 & .036 & \multicolumn{3}{|c|}{.02} & \multicolumn{2}{|c|}{.02} \\
\hline \multicolumn{2}{|l|}{ Capacidad predictiva (\%) } & $\begin{array}{l}\text { Global } \\
71.7 \\
\end{array}$ & & \multicolumn{2}{|c|}{$\begin{array}{c}\text { Baja frecuencia } \\
99.5\end{array}$} & \multicolumn{2}{|c|}{$\begin{array}{c}\text { Alta frecuencia } \\
1.4 \\
\end{array}$} \\
\hline Variables en la ecuación & $B$ & $E E$ & Wald & Gl & $P$ & $O R$ & $95 \%$ IC OR \\
\hline Energía & $-0.13^{*}$ & 0.06 & 4.31 & 1 & .03 & 0.88 & [0.78-0.99] \\
\hline
\end{tabular}

A través de las regresiones efectuadas, se observó que la energía predice la frecuencia de consumo actual y la frecuencia de CEEA. En el caso del consumo actual, el poder predictivo de la energía se mantiene al incorporar la variable edad en el modelo. La capacidad global del modelo para predecir frecuencia de consumo actual fue del $64 \%$, pudiendo clasificar correctamente al $91 \%$ de los sujetos con alta frecuencia y al $17.3 \%$ de los sujetos con baja frecuencia de consumo actual. La capacidad global del modelo para predecir frecuencia de CEEA fue

\section{DISCUSIÓN}

El presente estudio se inscribe en la línea de trabajos que contribuyen a un mayor conocimiento de los factores relacionados con la adopción de estilos de vida saludables. La actividad deportiva moderada posee efectos positivos innegables a nivel físico $\mathrm{y}$ psicológico, y es un importante medio de relación social. Sin embargo, la evidencia empírica actual no permite aseverar que la participación en actividades superior a la del modelo anterior (71.7\%), pudiendo clasificar correctamente casi la totalidad de casos con baja frecuencia de CEEA (99.5\%) y no pudiendo clasificar correctamente a casi ningún caso con alta frecuencia de CEEA (1.4\%). A pesar de ser buenos modelos para clasificar alguna de las condiciones, el coeficiente de determinación $(R)^{2}$ es bajo en ambos modelos. Esto indica que es poca la proporción de la variación que logran explicar. Ningún modelo resultó significativo para predecir la variable cantidad de consumo de alcohol (Tabla 3 ).

físico-deportivas per se constituya un factor protector del consumo de alcohol de los jóvenes. Siendo este último uno de los problemas de mayor impacto sanitario y social en este grupo etario, los esfuerzos por identificar las condiciones bajo las cuales el deporte podría disminuir o retrasar el comienzo del consumo, son ineludibles. 


\section{Compromiso con el deporte y su relación con el consumo de alcohol}

La Teoría de la Autodeterminación (Deci y Ryan, 1985; Ryan y Deci, 2000) ha abierto un camino promisorio para explorar los posibles efectos de las variables motivacionales sobre el consumo de alcohol. En las últimas décadas, han proliferado los estudios que muestran que la motivación intrínseca y las experiencias positivas con el deporte se encuentran asociadas a un menor consumo de alcohol (Rockafellow y Saules, 2006; Moreno-Murcia, González-Cutre Coll, y Cervello-Gimeno, 2008). Se sabe asimismo que la satisfacción de las necesidades psicológicas básicas y motivación intrínseca favorece, a su vez, el compromiso deportivo (Coterón, Sampedro, Franco y Pérez-Tejero, 2013; Hodge et. al., 2009). Además, otras investigaciones realizadas desde la Teoría de Metas de Logro respecto de la motivación indican que la orientación a la tarea predice positivamente la práctica de actividades deportivas y negativamente el consumo de sustancias perjudiciales para la salud, y que la orientación al ego no resulta ser un factor predictor de las conductas de salud (Balaguer, Castillo, Tomás y Duda, 1997). Al relacionar el estilo de vida saludable y las dimensiones del autoconcepto, se ha encontrado que los jóvenes que se autoperciben competentes en los deportes tienden a practicar deporte con mayor frecuencia. Es decir, cuanto mayor es la percepción de competencia deportiva, mayor es la práctica de deporte, y a mayor práctica de deporte, menor es el consumo de tabaco y de alcohol y mayor el consumo de alimentos sanos (Pastor, Balaguer y García-Merita, 2006). Pero el compromiso, como variable asociada a la práctica deportiva, no había sido analizado como posible factor protector en trabajos previos.

En el presente trabajo, se observó que las cuatro dimensiones del compromiso resultaron más altas en los grupos de menor frecuencia de consumo actual, menor cantidad de alcohol consumido y menor frecuencia de CEEA. Pero las diferencias sólo fueron significativas para el componente denominado energía y sólo considerando la frecuencia actual y frecuencia de CEEA. Es decir, quienes consumen de $\mathrm{Si}$ bien los efectos hallados son leves, resulta interesante que algún aspecto del compromiso sea predictor de la frecuencia de consumo. Cuando se trata de deporte colectivo, los estudios tienden a mostrar que las variables motivacionales no tienen efecto en el consumo o tienen efectos adversos. Es decir, algunos estudios encuentran que el deporte manera frecuente y quienes con frecuencia ingieren grandes cantidades de alcohol en la misma ocasión (CEEA), son quienes presentan menores niveles de energía. La cantidad de alcohol que consumen no se asocia a ningún componente del compromiso con el deporte.

Se probaron tres modelos de regresión, a través de los cuales se testeó la capacidad de del compromiso para predecir frecuencia, cantidad y frecuencia de CEEA. Se encontró que el compromiso, a través de su componente energía, predice la frecuencia de consumo actual y de CEEA. En el primer modelo, se observa que la energía mantiene su poder predictivo sobre la frecuencia de consumo actual aun cuando se incluye la variable edad. Si bien el porcentaje de varianza explicada con cada modelo (4 y $2 \%$ respectivamente) es bajo, se observan efectos significativos de la energía sobre la frecuencia de consumo actual y de la energía sobre la frecuencia de CEEA con una capacidad de aciertos relativamente adecuada (64\% y $71.7 \%$ respectivamente). Una baja energía conjuntamente con una mayor edad, permiten predecir la presencia de alta frecuencia de consumo actual con un $91 \%$ de los casos correctamente clasificados. Por su parte, una alta energía dedicada al deporte (independientemente de la edad y del género) permite estimar la baja frecuencia de CEEA con un $99.5 \%$ de aciertos. Ningún componente del compromiso predice la cantidad de alcohol ingerida en la misma ocasión. Es decir, no importa cuán comprometido esté el joven con su práctica deportiva, a la hora de consumir lo hará con la misma intensidad que un deportista no comprometido. Podría pensarse entonces que el componente energía del compromiso funcionaría como factor que protege del consumo frecuente de alcohol (sea moderado o excesivo) pero al tratarse de un estudio transversal, no es posible conocer la dirección de las relaciones. Quizá consumir alcohol atenta contra el compromiso (más puntualmente, contra la energía dedicada al deporte) o bien, la energía protege del consumo frecuente.

practicado en equipo se asocia a un mayor consumo de alcohol. En el estudio conducido por Rockafellow y Saules (2006) se encontró un sustento considerable para esta hipótesis y se concluyó que la participación en deportes colectivos se asocia a mayores tasas de consumo de sustancias, particularmente alcohol y tabaco. A su vez, Bedendo y Noto (2015) en su 


\section{Schmidt, V., Celsi, I., Molina, M.F., Raimundi, M.J., García-Arabehety,M., Pérez-Gaido, M., Iglesias, D., \& González, M.A.}

estudio con una amplia muestra de adolescentes brasileños encontraron que quienes practicaban deportes colectivos presentaban mayor consumo (frecuencia de consumo y CEEA) comparados con quienes no realizaban prácticas deportivas.

En el presente estudio, todos los sujetos de la muestra practican deporte colectivo y lo hacen en un contexto social en donde el consumo de alcohol está naturalizado y hasta valorado de manera positiva (SEDRONAR, 2017; Calero, Schmidt y Bugallo, 2016). Por lo tanto, es esperable encontrar lo que aquí se halló: que el consumo de alcohol del deportista no difiere del que realizan los jóvenes de población general. A las presiones ejercidas por el mercado de la bebida alcohólica a través de efectivas estrategias de marketing, se suma la presión ejercida por el grupo de pares. En esta etapa del desarrollo los jóvenes son particularmente sensibles a las recompensas cuando están en compañía de sus pares (Silva, Shulman, Chein, y Steinberg, 2016) y son más propensos que los adultos a tomar riesgos en el grupo de pares (Gardner y Steinberg, 2005). De hecho, la decisión de consumir grandes cantidades de alcohol muchas veces se asocia a la necesidad de pertenencia. En un estudio realizado en la Ciudad de Buenos Aires con adolescentes de población general, se ha encontrado que el miedo al rechazo (un componente de la necesidad de pertenencia) se asocia a mayor prevalencia de CEEA (Leibovich, Schmidt y Calero, 2018). Es en el grupo de pares donde las prácticas sociales asociadas al consumo excesivo cobran sentido. Se ha encontrado también en nuestro contexto que quienes más consumen reportan mayor aceptación social, atractivo amoroso y autoestima que aquellos que consumen de manera moderada o no consumen (Calero, Schmidt y Bugallo, 2016).

Es por ello que el resultado obtenido resulta valioso. Tal como ocurrió en estudios previos, dado el fuerte efecto que tiene la pertenencia al grupo de pares, se podría haber encontrado en este estudio que las variables de compromiso no se asocian al consumo de alcohol en estos jóvenes deportistas. De hecho, no función de variables relevantes tanto deportivas como sociodemográficas) con alta frecuencia de consumo actual a los que no se aplique intervención alguna. Otra posibilidad sería conducir un estudio con método mixto en el cual se incluyan, además de las variables estudiadas en el presente trabajo, estrategias cualitativas para indagar el sentido que los de alta frecuencia de consumo actual otorgan a los efectos se halló efecto sobre la cantidad de alcohol consumida lo que indica que, si bien la energía se asocia a una menor frecuencia, cuando consumen lo hacen en grandes cantidades.

Una cuestión interesante a indagar es la relación de la satisfacción de las necesidades psicológicas básicas con el compromiso como factor protector del consumo. Hogde et al. (2009) encontraron que necesidad de autonomía y competencia, pero no la de relación (pertenencia) eran predictores positivos del compromiso en deportistas de elite. En el presente estudio no se ha analizado el grado de pertenencia social dentro del equipo o del deporte, pero podría pensarse que en deportistas de un nivel competitivo menor (no alto rendimiento), la necesidad de pertenencia (relación) puede asociarse a un mayor compromiso y éste vincularse a un menor consumo de alcohol.

En síntesis, el resultado obtenido en la presente investigación permite concluir que la energía dedicada al deporte tiene relación con la frecuencia de consumo, si bien la actividad se realiza con grupo de pares y en un entorno que alienta el consumo. Pero al mismo tiempo, el bajo porcentaje de varianza explicado por ambos modelos indica que existen otras variables que tienen efectos sobre la probabilidad de consumir de manera frecuente alcohol. En el futuro sería interesante para lograr obtener modelos con mayor capacidad predictiva incluir, además de las variables motivacionales asociadas al deporte, características de personalidad y del entorno que se hallan fuertemente asociadas al consumo.

Por otra parte, en futuros estudios será necesario profundizar en el análisis de estas relaciones tratando de conocer la dirección de las mismas o el sentido que otorgan los sujetos a las relaciones halladas. Se podría llevar a cabo una intervención para aumentar el nivel de compromiso en el grupo con alta frecuencia de consumo (moderado o excesivo) y estudiar sus efectos, contando con un grupo control (equiparado en que el alcohol tiene sobre su práctica y sobre la energía invertida, así como conocer los motivos de quienes suelen evitar el consumo excesivo en el contexto cotidiano de su práctica deportiva.

Respecto de las variables sociodemográficas indagadas (género y edad), en coincidencia con los últimos estudios epidemiológicos (SEDRONAR, 2017; OPS, 2015), en el presente estudio se observa 


\section{Compromiso con el deporte y su relación con el consumo de alcohol}

que la variable género no tiene efectos en la frecuencia y cantidad de alcohol consumida (ambos, varones y mujeres consumen de manera similar). Respecto de la variable edad, se observa que es buen predictor de una de las variables dependientes: la frecuencia de consumo actual. En muchos estudios (p. ej., Castaño Ruiz, 2014) se advierte la importancia de considerar el fenómeno del consumo de sustancias desde una perspectiva de género superadora de la lógica binaria. Por este motivo, en futuros estudios, sería interesante seguir profundizando en los efectos del género sobre las variables bajo estudio. En algunos estudios con muestras de deportistas que han contemplado la variable género, se encontraron resultados diferenciales. Por ejemplo, en jóvenes deportistas de Argentina el apoyo a la autonomía por parte del entrenador tiene mayor influencia en el compromiso de los varones que en el de las mujeres, pero mayor influencia en la intención de abandono del deporte en éstas últimas, en comparación con los varones (Raimundi, García-Arabehety, Iglesias, Pérez-Gaido, Morillo y Schmidt, 2017).

\section{APLICACIONES PRÁCTICAS}

Los aportes de la SDT han sido de gran utilidad para elaborar diferentes intervenciones en el campo de la promoción de la salud, pero aún son escasos los estudios que aborden esta cuestión específica relativa al consumo de alcohol (Smith, 2011). Sin embargo, es posible pensar estrategias específicas desde los programas que vienen realizándose en el campo del deporte de manera eficaz a través del trabajo directo con los deportistas para favorecer implicaciones positivas desde la motivación autodeterminada, la pasión, las aspiraciones vitales o el compromiso con los jóvenes deportistas (Raimundi, García-Arabehety, Iglesias y Castillo, 2019) o a través del trabajo indirecto a través de los agentes sociales (entrenadores y padres). Programas de formación tales como Empowering Coaching ${ }^{\mathrm{TM}}$ (Duda, 2013), Escuelas de padres y madres (Gimeno, 2007), Psytool (García-Mas, Rosado, Serpa, Marcolino y Villalonga, 2018) son ejemplos concretos de intervenciones indirectas para favorecer experiencias positivas en el deporte formativo y así generar un contexto de desarrollo y bienestar que contribuya a la protección de conductas de riesgo como el consumo de alcohol.

\section{CONCLUSIONES}

En síntesis, los deportistas están inmersos en el mismo contexto de influencias que los no deportistas y de allí que no haya diferencias importantes en la modalidad de consumo. Sin embargo, el resultado del presente estudio es alentador en tanto aquellos jóvenes que logran comprometerse con la actividad deportiva presentan menor frecuencia de consumo actual y de CEEA. Trabajar para facilitar el compromiso con el deporte podría ser una de las estrategias posibles para aumentar los efectos protectores de la práctica deportiva sobre el consumo de alcohol.

\section{AGRADECIMIENTOS}

A los deportistas que participaron del estudio, a sus entrenadores y a los clubes que abrieron sus puertas: Ferro Carril Oeste, Club Ciudad de Buenos Aires, Club Banco Ciudad, Club Atlético Banco de la Nación Argentina, Atlético San Andrés Rugby Club. A los organismos que financiaron el proyecto: Secretaría de Ciencia y Técnica de la Universidad de Buenos Aires (Proyecto UBACyT 2018-2000 Consumo de alcohol en población joven: factores protectores a través del deporte y la música, director: Schmidt, V.; co-director: Leibovich, N.). Consejo Nacional de Investigaciones Científicas y Técnicas (Proyecto PIP de CONICET: Motivación y compromiso con la actividad físico-deportiva como factores protectores del consumo de sustancias psicoactivas en población joven, director: Schmidt, V.; co-director: Leibovich, N.). Universidad Abierta Interamericana (Proyecto UAI 2016-2018: Consumo de sustancias psicoactivas en escenarios nocturnos de presencia masiva. Su relación con prácticas de riesgo $\mathrm{y}$ de cuidado en población universitaria, director: Schmidt, V.; co-director: Raimundi, M.J.).

\section{REFERENCIAS}

1. Balaguer, I., Castillo, I., Tomás, I., y Duda, J.L. (1997). Las orientaciones de metas de logro como predictoras de las conductas de salud en los adolescentes. Iber Psicología, 2, 2-10.

2. Balaguer, I. (Ed.). (2002). Estilos de vida en la adolescencia. Valencia: Promolibro. 


\section{Schmidt, V., Celsi, I., Molina, M.F., Raimundi, M.J., García-Arabehety,M., Pérez-Gaido, M., Iglesias, D., \& González, M.A.}

3. Balaguer, I., Atienza, F., y Duda, J. (2012). Selfperceptions, self-worth and sport participation in adolescents. The Spanish Journal of Psychology, 15(2),624-630.

http://doi.org/10.5209/rev_SJOP.2012.v15.n2.38 $\underline{873}$

4. Balaguer, Castillo y Duda (2008). Apoyo a la autonomía, satisfacción de las necesidades, motivación y bienestar en deportistas de competición: Un análisis de la Teoría de la Autodeterminación. Revista de Psicología del Deporte, 17(1), 123-139.

5. Bedendo, A. y Noto, A. R. (2015) Sports practices related to alcohol and tobacco use among high school students. Revista Brasileira de Psiquiatria, 37, 99-105. http://doi.org/10.1590/1516-4446-2014-1389

6. Bewick, V., Cheek, L., y Ball, J. (2005). Statistics review 14: Logistic regression. Critical Care, 9(1), 112-118. http://doi.org/10.1186/cc3045

7. Bush, K.; Kivlahan, D.R.; McDonell, M.B.; Fihn, S.D. y Bradley, K.A. (1998). The AUDIT alcohol consumption questions (AUDIT-C): an effective screening test for problem drinking. Ambulatory Care Quality Improvement Project (ACQUIP). Alcohol Use Disorders Identification Test. Archive Internal Medicine, 158(16), 1789-1795. http://doi.org/10.1001/archinte.158.16.1789

8. Calero, A.; Schmidt, V. y Bugallo, L. (2016). Consumo de alcohol y su relación con la autopercepción adolescente. Health and Addictions, $16(1), \quad$ 49-58. http://doi.org/10.21134/haaj.v16i1.259

9. Castaño Ruiz, V. (2014). Diferencias en la percepción de consumo recreativo de drogas entre chicos y chicas jóvenes. Un análisis desde la perspectiva de género. Madrid: Fundación Atenea. Recuperado de http://fundacionatenea.org/http:/fundacionaten ea.org/wpcontent/uploads/2014/11/ChicasyChicosConsum Recreat FundAteneaPNSD 2014-2.pdf

10. Castillo, I., Balaguer, I., y García-Merita, M. (2007). Efecto de la práctica de actividad física y de la participación deportiva sobre el estilo de vida saludable en la adolescencia en función del género. Revista de Psicología del Deporte, 16, 201-210.
11. Ceschini, F. L., Luiz de Andrade, E., y Figueira, A. (2015). Physical activity and associated factors among students attending evening classes. Revista Brasileira de Cineantrometria e Desempenho Humano, 17(2), 205-215. http://doi.org/10.5007/1980-037.2015v17n2p205

12. Conde, K.; Gimenez, P., y Cremonte, M. (2018). Características de los instrumentos de tamizaje del trastorno por uso de alcohol en jóvenes universitarios de Argentina. Revista Clínica y Salud, 29, 27-33.

13. Coterón, J., Sampedro, J., Franco, E., PérezTejero, J. y Refoyo, I. (2013). The role of basic psychological needs in predicting dispositional flow of basketball players in training. Differences by sex. Revista de Psicología del Deporte, 22(1), 187-190.

14. Cremonte, M., Ledesma, R.D., Cherpitel, C.J., y Borges, G. (2010). Psychometric properties of alcohol screening tests in the emergency department in Argentina, Mexico and the United States. Addictive Behaviors, 35(9), 818-825. http://doi.org/10.1016/j.addbeh

15. Deci, E. (1975). Intrinsic motivation. New York: Plenum.

16. Deci, E. L., y Ryan, R. M. (1985). The general causality orientations scale: Self-determination in personality. Journal of Research in Personality, 19, 109-134.

17. Duda, J. (2013). The conceptual and empirical foundations of Empowering Coaching TM: Setting the stage for the PAPA project. International Journal of Sport and Exercise Psychology, 11(4), 37-41. http://doi.org/10.1080/1612197X.2013.839414

18. Ferriz, R. (2014). Importancia de la satisfacción en las clases de educación fisica para la motivación y la adopción de un estilo de vida saludable. Universidad de Almería, Almería.

19. García-Mas, A., Rosado, A., Serpa, S., Marcolino, P., y Villalonga, C. (2018). Content analysis of the agents of change "disposition to change" after attending the Psytool Program. Revista de Psicología del Deporte, 27(3), 31-36.

20. Gardner, M., y Steinberg, L. (2005). Peer Influence on Risk Taking, Risk Preference, and Risky Decision Making in Adolescence and Adulthood: An Experimental Study. Developmental Psychology, 41(4), 625-635. http://doi.org/10.1037/0012-1649.41.4.625 


\section{Compromiso con el deporte y su relación con el consumo de alcohol}

21. Gimeno, F. (2007). Descripción y evaluación preliminar de un programa de habilidades sociales y de solución de problemas con padres y entrenadores en el deporte infantil y juvenil. Revista de Psicología del Deporte, 12(1), 67-79.

22. Hedstrom, R., y Gould, D. (2004). Research in youth sports: Critical issues status. Michigan: Michigan State University, 1-42.

23. Hernández Sampieri, R; Fernández-Collado, C., y Baptista Lucio, M.P. (2014). Metodología de la Investigación. México: McGrawHill/Interamericana Editores.

24. Hodge, K., Lonsdale, C., y Jackson, S. (2009). Athlete engagement in elite sport: An exploratory investigation of antecedents and consequences. The Sport Psychologist, 23(1), 186-202. http://doi.org/10.1123/tsp.23.2.186

25. Jiménez, M.G., Martínez, P., Miró, E., y Sánchez A.I. (2008). Bienestar psicológico y hábitos saludables: ¿están asociados a la práctica de ejercicio físico? International Journal of Clinical and Health Psychology, 8(1), 185-202.

26. Jones, D. N., y Paulhus, D. L. (2011). The role of impulsivity in the Dark Triad of personality. Personality and Individual Differences, 51(5), 679-682.

http://doi.org/10.1016/j.paid.2011.04.011

27. Latorre Román, P. A., Cámara Pérez, J. C., y García Pinillos, F. (2014). Búsqueda de sensaciones y hábitos de tabaquismo, consumo de alcohol y práctica deportiva en estudiantes de Educación Secundaria. Salud Mental, 37(2), 145152. $\quad$ http://doi.org/10.17711/SM.0185$\underline{3325.2014 .018}$

28. Leibovich, N.; Schmidt, V., y Calero, A. (2018). The Need to Belong (NB) in Adolescence: Adaptation of a Scale for its Assessment. Psychology and Behavioral Science, 8(5), 001007.

http://doi.org/10.19080/PBSIJ.2018.08.555747

29. Lonsdale, C., Hodge, K., y Jackson, S. (2007). Athlete engagement: II. Development and initial validation of the Athlete Engagement Questionnaire. International Journal of Sport Psychology, 38, 471-492.

30. Lorente, F.O.; Souville, M.; Griffet, J., y Grélot, L. (2004). Participation in sports and alcohol consumption among French adolescents. Addictive Behaviors, 29(5), 941-946. http://doi.org/10.1016/j.addbeh.2004.02.039
31. Moore, M. J. (2005). Sport and physical activity participation and substance use among adolescents. Journal of Adolescent Health, 36, 486-493.

http://doi.org/10.1016/j.jadohealth.2004.02.031

32. Moreno-Murcia, J.A.; González-Cutre Coll, D., y Cervello-Gimeno, E. (2008). Motivación y salud en la práctica físico-deportiva: diferencias según el consumo de alcohol y tabaco. International Journal of Clinical and Health Psychology, 8(2), 483-494.

33. Observatorio Argentino de Drogas, OAD (2014). Sexto estudio nacional sobre consumo de sustancias psicoactivas en estudiantes de enseñanza media. Buenos Aires: SEDRONAR.

34. Observatorio Argentino de Drogas, OAD (2017a). Mortalidad relacionada al consumo de sustancias psicoactivas. Argentina 2015. Buenos Aires: SEDRONAR.

35. Observatorio Argentino de Drogas, OAD (2017b). Análisis de los contextos individuales y sociofamiliares en jóvenes escolarizados y su relación con el consumo de alcohol y marihuana. Buenos Aires: SEDRONAR.

36. Oficina de las Naciones Unidas contra la Droga y el Delito (2014). Actividades alternativas para la prevención del uso de drogas. Quito: UNODC.

37. Organización Mundial de la Salud, OMS (2004) Informe sobre la salud en el mundo 2004 cambiemos el rumbo de la historia. Ginebra, Suiza.

38. Organización Mundial de la Salud, OMS (2010). Estrategia mundial para reducir el uso nocivo de alcohol. Italia: WHO.

39. Organización Mundial de la Salud, OMS (2014). Global Status Report on Alcohol and Health. Geneva: WHO.

40. Organización Panamericana de la Salud, OPS (2010). Salud y bienestar de los adolescentes y jóvenes: una mirada integral. Washington, D.C.: WHO.

41. Organización Panamericana de la Salud, OPS (2015). Regional Status Report on Alcohol and Health in the Americas. Washington, D.C.: WHO.

42. Pastor, Y., Balaguer, I., y García-Merita, M. (2006). Relaciones entre el autoconcepto y el estilo de vida saludable en la adolescencia media: un modelo exploratorio. Psicothema, 18(1), 1824. 


\section{Schmidt, V., Celsi, I., Molina, M.F., Raimundi, M.J., García-Arabehety,M., Pérez-Gaido, M., Iglesias, D., \& González, M.A.}

43. Peng, C.-Y. J., Lee, K. L., y Ingersoll, G. M. (2002). An introduction to logistic regression analysis and reporting. The Journal of Educational Research, 96(1), 3-14. http://doi.org/10.1080/00220670209598786

44. Raimundi, M. J., García-Arabehety, M., Iglesias, D. H. y Castillo, I. (2019). Aspiraciones vitales y su relación con la pasión en deportistas argentinos seleccionados para los Juegos Olímpicos de la Juventud. Cuadernos de Psicología del Deporte, 19(1), 192-205.

45. Raimundi, M. J., García-Arabehety, M., Iglesias, D. H., Pérez-Gaido, M., Morillo, M., y Schmidt, V. (2017). The influence of motivational climate in engagement of Argentinean athletes from Youth Olympic Games. 14th World Congress of Sport Psychology, Sevilla.

46. Rockafellow, B. D., y Saules, K. K. (2006). Substance use by college students: The role of intrinsic versus extrinsic motivation for athletic involvement. Psychology of Addictive Behaviors, 20(3), 279-287. http://doi.org/10.1037/0893164X.20.3.279

47. Ruiz-Juan, F., y Ruiz- Risueño, J. (2011). Variables predictoras de consumo de alcohol entre adolescentes españoles. Anales de Psicología, 27(2), 350-359.

48. Ryan, R., y Deci, E. (2000). Self-determination Theory and the facilitation of intrinsic motivation, social development and well-being. American Psychologist, 55(1), 68-78. http://doi.org/10.1037110003-066X.55.1.68

49. Saunders, J., Aasland, O., Babor, T., De la Fuente, J., y Grant, M. (1993). Development of the Alcohol Use Disorders Identification Test (AUDIT): Who Collaborative Project on Early Detection of Persons with Harmful Alcohol Consumption - II. Addiction, 88(6), 791-804.

50. Secretaría de Políticas Integrales sobre Drogas de la Nación Argentina, SEDRONAR (2017a). Tabaco-Alcohol. Intensidad de consumo. Estudio Nacional en población de 12 a 65 años, sobre Consumo de Sustancias Psicoactivas. Argentina 2017. Presidencia de la Nación Argentina.

51. Secretaría de Políticas Integrales sobre Drogas de la Nación Argentina, SEDRONAR (2017b). Estudio Nacional en población de 12 a 65 años, sobre Consumo de Sustancias Psicoactivas. Argentina 2017. Presidencia de la Nación Argentina.
52. Secretaría de Políticas Integrales sobre Drogas de la Nación Argentina, SEDRONAR (2017c). Resumen de los Resultados del Estudio 2017 de Consumo de Sustancias Psicoactivas. Presidencia de la Nación Argentina.

53. Silva, K., Shulman, E. P., Chein, J., y Steinberg, L. (2016). Peers Increase Late Adolescents' Exploratory Behavior and Sensitivity to Positive and Negative Feedback. Journal of Research on Adolescence, 26(4), 696-705. http://doi.org/10.1111/jora.12219

54. Smith, L. (2011). Self Determination Theory and potential applications to alcohol and drug abuse behaviors. Journal of Alcohol and Drug Education, 55, 3-7.

55. Usán, P., y Salavera, C. (2017). Influencia de la motivación hacia el deporte en el consumo de alcohol, tabaco y cannabis de adolescentes escolares. Actualidades en Psicología, 31(122), 119-131. http://dx.doi.org/10.15517/ap.v31i122.27980

56. Vaquero-Cristóbal, R., Isorna, M., y Ruiz, C. (2012). Development of the Alcohol Use Disorders Identification Test. Journal of Sport and Health Research, 4(3), 269-288. 